the oocyte cytoplasm. Conclusive determination of which proteins are bound to osk mRNA at each step of this complex journey will require new approaches. Biochemical extracts cannot detect subtle changes in the components that are bound to an mRNA at distinct steps of its localization, and immunolocalization or green fluorescent protein (GFP) fusion data cannot exclude the possibility that a protein present at levels below the detection threshold is required. Investigating the subtle changes in RNP composition within living cells is likely to require the ability to detect rapidly changing physical interactions between single protein and mRNA molecules using new and emerging imaging technologies ${ }^{14}$.

Nonetheless, Palacios et al. have shown that not only are nuclear EJC components required for osk localization in the cytoplasm, but also that a putative RNA helicase and translational regulation factor that functions in NMD in humans is also required for osk mRNA localization. This raises many exciting questions. For example, is the RNA helicase activity of eIF4AIII required to remodel RNP composition? Could eIF4AIII be involved in translational inhibition of osk mRNA while it is being transported to the posterior cytoplasm? Is eIF4AIII required for the localization of other transcripts in flies and human neurons? Whatever the answers to these questions, it is probable that even more trans-acting factors with diverse roles in RNA biogenesis will be required for osk mRNA localization and it is unlikely that the specificity of osk mRNA sorting in the cytoplasm could be provided by a single factor. Instead, mRNA localization in the oocyte is likely to be just as complex as mRNA processing and export, or most other RNA-based processes $^{12,13}$. In the final analysis, cytoplasmic destination may be selected by many factors binding RNA indiscriminately with specificity being provided by the overall geometry of a complex.

1. Ephrussi, A. \& St Johnston, D. Cell 116, 143-152 (2004).

2. Ephrussi, A. \& Lehmann, R. Nature 358, 387-392 (1992).

3. Tekotte, H. \& Davis, I. Trends Genet. 18, 636-642 (2002).

4. MacDougall, N., Clark, A., MacDougall, E. \& Davis, I. Dev. Cell 4, 307-319 (2003).

5. Brendza, R. P., Serbus, L. R., Duffy, J. B. \& Saxton, W. M. Science 289, 2120-2122 (2000).

6. Palacios, I. M., Gatfield, D., St Johnston, D. \& Izaurralde, E. Nature 427, 753-757 (2004).

7. Jansen, R. P. Nature Rev. Mol. Cell Biol. 2, 247-256 (2001).

8. Arn, E. A., Cha, B. J., Theurkauf, W. E. \& Macdonald, P. M. Dev. Cell 4, 41-51 (2003).

9. Palacios, I. M. Curr. Biol. 12, R50-R52 (2002).

10. Chan, C. C. et al. RNA 10, 200-209 (2004).

11. Gatfield, D., Unterholzner, L., Ciccarelli, F. D., Bork, P. \& Izaurralde, E. EMBO J. 22, 3960-3970 (2003).

12. Stutz, F. \& Izaurralde, E. Trends Cell Biol. 13, 319-327 (2003).

13. Mitchell, P. \& Tollervey, D. Curr. Opin. Cell Biol. 13, 320-325 (2001).

14. Tsien, R. Y. Nature Rev. Mol. Cell Biol. 4, Suppl, SS16-SS21 (2003).

\section{New neurons?}

The identification and characterization of neural stem cells within different regions of the adult brain, particularly in rodents, has revolutionized our understanding of adult neurogenesis and heightened hopes for treatments of traumatic damage and neurodegenerative disease. However, the degree to which this occurs in the adult human central nervous system has been less well characterized. Recently, Avlarez-Buylla and colleagues (Nature 427, 740-744 (2004)) identified some similarities, as well as some striking species-specific differences, for a pool of neural stem cells that reside in a specialized region of the human sub-ventricular zone (SVZ) - a part of the forebrain that lines the ventricular surface.

In mammals, the adult brain has two main areas that produce precursors for the generation of new neurons: the SVZ and the dentate gyrus of the hippocampus. In rodents, the SVZ produces a train of chain-migrating, newly formed neurons - the rostral migratory stream - that replace a population of inter-neurons in the olfactory bulb. Although human brains were known to contain neural precursors, the precise anatomical localization of these precursors and the degree to which they produce new neurons (particularly outside the dentate gyrus) remained relatively unclear.

Now, Alvarez-Buylla and colleagues use large numbers of cells from human biopsy and autopsy tissue samples to characterize the human neural stem cell pool. They find that the stem cells are localized to a ribbon-like layer of astrocytes lining the inner SVZ; this pronounced layer of the SVZ has not been identified in other mammalian species investigated. This population of astrocytes contained a small proportion $(\sim 4 \%)$ of cells that could be clonally expanded to produce both neuronal and glial cell lineages. Thus, it is clear that the SVZ in humans contains potential neural stem cells. However, the authors find no strong evidence to

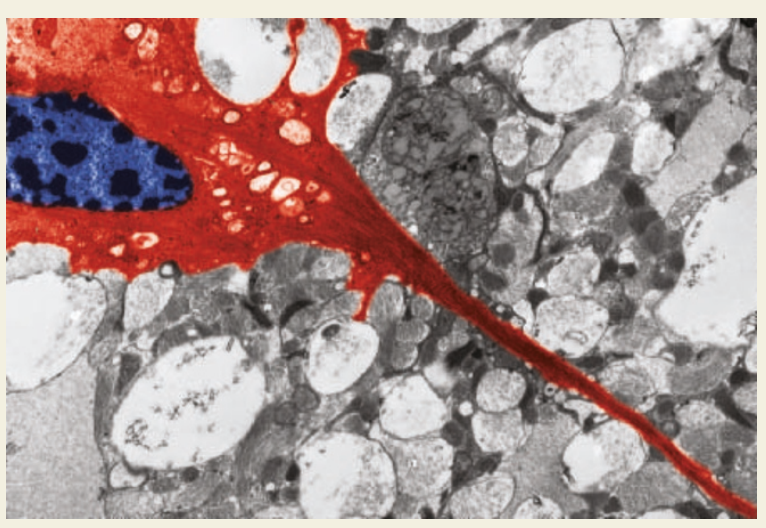

An electron micrograph of single astrocyte (blue nucleus) within the adult human SVZ astrocyte ribbon, which is extending a process of intermediate filaments (red) through the 'gap' region towards the ependyma.

suggest that that these cells are producing differentiated neurons in vivo. Most strikingly, they seem to completely lack the rostral migratory stream that is so prominent in rodents and that has even been identified in primate species.

An important conclusion that can be drawn from this work is that although humans clearly possess neural precursor cells that could potentially be used to replace areas of damaged neurons, the situation in adult humans is clearly different from that in other mammalian species. These differences have obvious implications for interpreting animal studies as a basis for human therapies. Uncovering the molecular underpinning of these differences will be necessary for deriving successful cell-replacement treatments for traumatic and neurodegenerative brain disorders.

JACK HORNE 